\title{
The mechanism of formate oxidation by metal-dependent formate dehydrogenases
}

\author{
Cristiano S. Mota - Maria G. Rivas · Carlos D. Brondino • Isabel Moura • \\ José J. G. Moura · Pablo J. González • Nuno M. F. S. A. Cerqueira
}

Received: 7 February 2011/ Accepted: 20 June 2011/Published online: 20 July 2011

(C) SBIC 2011

\begin{abstract}
Metal-dependent formate dehydrogenases (Fdh) from prokaryotic organisms are members of the dimethyl sulfoxide reductase family of mononuclear molybdenum-containing and tungsten-containing enzymes. Fdhs catalyze the oxidation of the formate anion to carbon dioxide in a redox reaction that involves the transfer of two electrons from the substrate to the active site. The active site in the oxidized state comprises a hexacoordinated molybdenum or tungsten ion in a distorted trigonal prismatic geometry. Using this structural model, we calculated the catalytic mechanism of Fdh through density functional
\end{abstract}

Electronic supplementary material The online version of this article (doi:10.1007/s00775-011-0813-8) contains supplementary material, which is available to authorized users.

C. S. Mota - M. G. Rivas - I. Moura · J. J. G. Moura (凹) ·

P. J. González ( $\square)$

REQUIMTE, Departamento de Química,

Centro de Química Fina e Biotecnologia,

Faculdade de Ciências e Tecnologia,

Universidade Nova de Lisboa,

2829-516 Caparica, Portugal

e-mail: jose.moura@dq.fct.unl.pt

P. J. González

e-mail: pablo.gonzalez@dq.fct.unl.pt

C. D. Brondino

Departamento de Física,

Facultad de Bioquímica y Ciencias Biológicas,

Universidad Nacional del Litoral,

S3000ZAA, Santa Fe, Argentina

N. M. F. S. A. Cerqueira ( $\square)$

REQUIMTE, Departamento de Química,

Faculdade de Ciências, Universidade do Porto,

Rua do Campo Alegre s/n, 4169-007 Porto, Portugal

e-mail: nscerque@fc.up.pt theory tools. The simulated mechanism was correlated with the experimental kinetic properties of three different Fdhs isolated from three different Desulfovibrio species. Our studies indicate that the $\mathrm{C}-\mathrm{H}$ bond break is an event involved in the rate-limiting step of the catalytic cycle. The role in catalysis of conserved amino acid residues involved in metal coordination and near the metal active site is discussed on the basis of experimental and theoretical results.

Keywords Formate dehydrogenase - Reaction mechanism · Molybdenum $\cdot$ Tungsten $\cdot$ Density functional theory
Abbreviations
DFT
Density functional theory
ES
Enzyme-substrate
Fdh Formate dehydrogenase
PDB Protein Data Bank
SeCys Selenocysteine
$\mathrm{S}_{\mathrm{i}} \quad$ Inorganic sulfur atom
Tris-HCl Tris(hydroxymethyl)aminomethane
hydrochloride

\section{Introduction}

Formate dehydrogenases (Fdhs) catalyze the oxidation of formate to carbon dioxide according to the reaction [1]

$\mathrm{HCOO}^{-} \rightarrow \mathrm{CO}_{2}+\mathrm{H}^{+}+2 e^{-} \quad E^{\circ}=-461 \mathrm{mV}(\mathrm{pH} 8.00)$.

In anaerobic prokaryotes, the oxidation of formate is used as the main electron source for a variety of pathways that use as the final electron acceptor molecules other than 
dioxygen. Formate oxidation might also be part of a mechanism to form a proton gradient, once small uncharged molecules such as formic acid can cross the cytoplasmic membrane to the periplasmic space and release two protons after oxidation [2, 3].

Prokaryote Fdhs are metalloenzymes with different subunit composition containing either molybdenum or tungsten at the active site. The crystal structures of three of these enzymes have been reported. The molybdenumcontaining enzymes are Fdh-H and the membrane-bound Fdh-N from Escherichia coli K12 [4-10]. The tungstencontaining enzyme is the periplasmic Fdh from the sulfatereducing bacterium Desulfovibrio gigas [9-12]. The large subunit of these three enzymes $(80-110 \mathrm{kDa})$ contains the active site and one electron transfer center of the type [4Fe-4S]. In addition, they share high primary sequence identity and have a very similar polypeptide fold [9]. In the oxidized state, the active site contains either a Mo(VI) or a $\mathrm{W}(\mathrm{VI})$ ion hexacoordinated in a distorted trigonal prismatic geometry to four sulfur atoms from two pyranopterin molecules, one selenium atom from a selenocysteine (SeCys), and one inorganic sulfur atom, hereafter called $\mathrm{S}_{\mathrm{i}}$ (Fig. 1a). Two conserved arginine and histidine residues close to the metal ion, although not involved in metal coordination, were suggested to have key roles in catalysis $[4,13]$. In contrast, the crystallographic structure of the reduced molybdenum-containing E. coli Fdh-H (i.e., enzyme incubated with the substrate under anaerobic conditions) shows a pentacoordinated molybdenum ion in a distorted square-pyramidal geometry, in which the four sulfur atoms from the two pyranopterin molecules occupy the vertices of the equatorial plane and the $S_{i}$ ligand is in the apical position (Fig. 1b) [13]. The $S_{i}$ ligand was firstly proposed to be an oxygenic species [4] but was later identified as a sulfur atom from structural studies of D. gigas tungsten-containing Fdh and the reevaluation of the crystallographic data of $E$. coli $\mathrm{Fdh}-\mathrm{H}$ in the reduced state $[12,13]$. The latter also showed that the SeCys ligand is not coordinated to the molybdenum ion in the reduced form of the enzyme (Fig. 1b). Although the structure of a reduced $\mathrm{Fdh}$ was only determined for E. coli Fdh-H, spectroscopic studies performed on the Fdh from Desulfovibrio desulfuricans ATCC 27774 suggest that all molybdenum-dependent Fdhs have similar characteristics in their oxidized and reduced states [14, 15].

Kinetic and spectroscopic data of E. coli Fdh-H showed that the oxidation of formate implies the cleavage of the $\mathrm{C}-\mathrm{H}$ bond to give a $\mathrm{CO}_{2}$ molecule, and that the $\alpha$ proton of the substrate is transferred to an acceptor group located near the molybdenum ion $[4,5]$. On this basis and from structural data, two rather different mechanisms have been proposed by Boyington et al. [4] and Raaijmakers and Romao [13]. Both proposals involve the binding of the formate molecule to the molybdenum ion and the SeCys residue as the proton acceptor group, but differ essentially in how the molybdenum-formate interaction occurs. The formate-active site interaction in the proposal by Raaijmakers and Romao, which is based on the structures depicted in Fig. 1, occurs after breaking of the Se-Mo bond, with the $S_{i}$ ligand being coordinated to molybdenum along the catalytic cycle. In contrast, the leaving group in the mechanism of Boyington et al. is an $\mathrm{OH}$ ligand, now
Fig. 1 a Coordination around the metal ion of oxidized molybdenum/tungstencontaining formate dehydrogenase (Fdhs) [Protein Data Bank (PDB) ID 1H0H or 1FDO]. $\mathbf{b}$ Reduced form of Escherichia coli Fdh-H (PDB ID 2IV2). Conserved residues of selenocysteine (Se-cysteine), histidine, and arginine are also included. $S_{i}$ inorganic sulfur atom
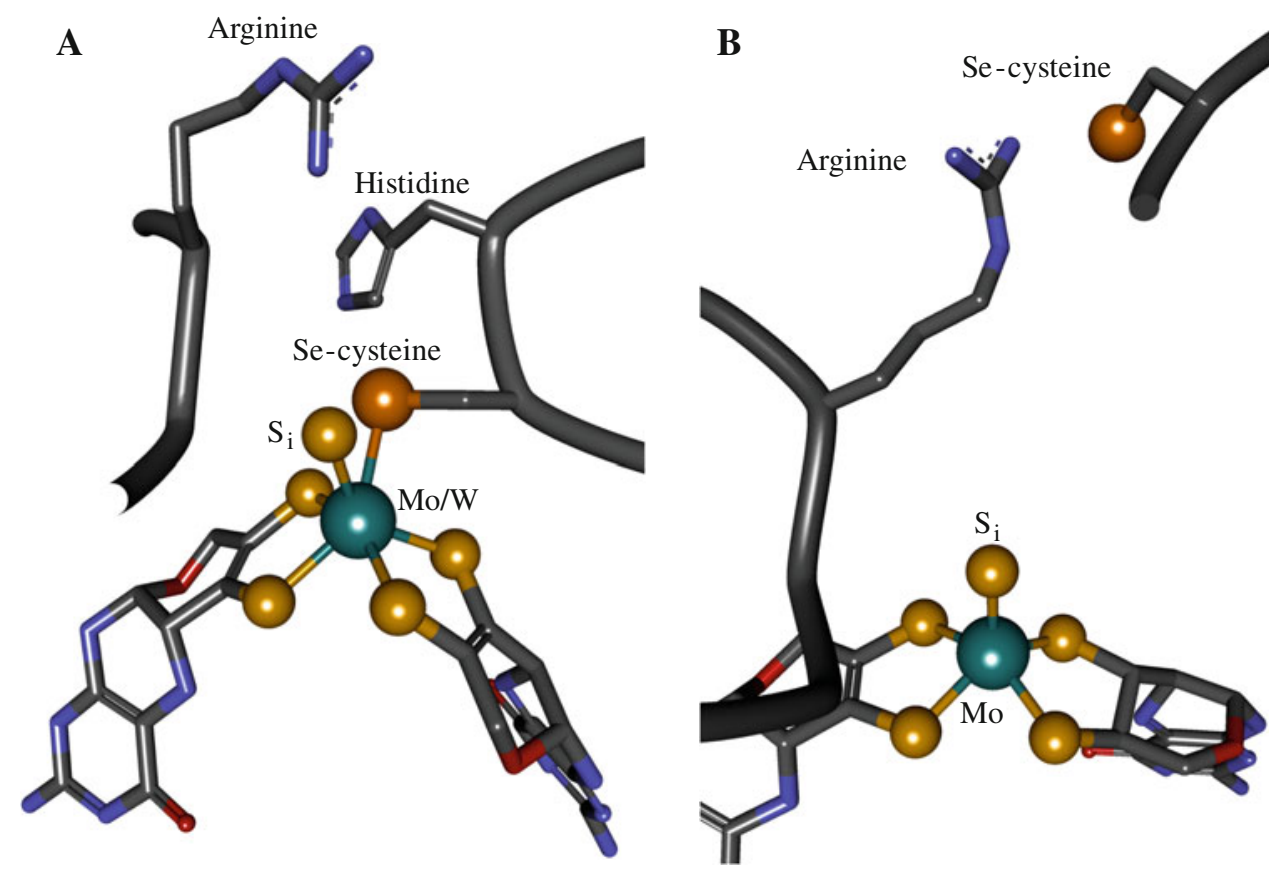
assigned to the $S_{i}$ ligand (Fig. 1a), and the Se ligand is coordinated to molybdenum along the entire catalytic cycle [4]. Both mechanisms were assessed by Leopoldini et al. [16] using theoretical and computational means. These authors concluded that the SeCys residue is the protonaccepting group and that the mechanism of Raaijmakers and Romao is kinetically and thermodynamically more favorable than that of Boyington et al. (activation energies of 19 and $36 \mathrm{kcal} / \mathrm{mol}$, respectively) [16]. However, Leopoldini et al. only studied the proton abstraction reaction, and avoided in their analysis essential aspects for the catalysis such as the role of the conserved histidine and arginine residues, as well as the processes of substrate binding and product release.

In the work reported here, the catalytic mechanism of Fdh was simulated using density functional theory (DFT) tools and correlated with experimental kinetic data obtained in three Fdhs isolated from Desulfovibrio species. This procedure allowed us to address how the enzymesubstrate (ES) interaction occurs, the role of the amino acid residues close to the active site, the conformational changes experienced by the metal site, and the relevance of the metal ions molybdenum and tungsten in the catalysis.

\section{Materials and methods}

Bacterial strain, culture media, and growth conditions

D. desulfuricans ATCC 27774, D. gigas NCIB 9332, and Desulfovibrio alaskensis cells were, respectively, grown in lactate-nitrate [17], lactate-sulfate [18], and in a modified medium $\mathrm{C}$ from Postgate [19]. Cells were grown under anaerobic conditions at $37{ }^{\circ} \mathrm{C}$ and collected by centrifugation at the end of the exponential phase.

\section{Enzyme purification}

Molybdenum-containing Fdh from D. desulfuricans ATCC 27774 was purified as described by Rivas et al. [15]. Tungsten-containing Fdh from D. alaskensis was purified as described by Mota et al. [19]. Tungsten-containing Fdh from $D$. gigas was purified with a method different from that reported before [20]. The soluble extract was loaded onto a column with DE-52 resin (Whatman) equilibrated with $10 \mathrm{mM}$ tris(hydroxymethyl)aminomethane hydrochloride (Tris- $\mathrm{HCl}$ ). Elution was performed using a linear gradient from 10 to $500 \mathrm{mM}$ Tris- $\mathrm{HCl}$ in 5 column volumes. The fractions containing Fdh were dialyzed and loaded onto a Source 15Q column (GE Healthcare) equilibrated with $5 \mathrm{mM}$ Tris- $\mathrm{HCl}$ and were eluted using a linear gradient until $300 \mathrm{mM}$ Tris- $\mathrm{HCl}$ in 10 column volumes. The fractions containing Fdh activity were pooled and loaded onto a hydroxyapatite column equilibrated with $1 \mathrm{mM}$ potassium phosphate buffer and were eluted using a linear gradient until $300 \mathrm{mM}$ potassium phosphate buffer in 10 column volumes. Finally, the fraction containing Fdh was concentrated by ultrafiltration in a Centricon system (Amicon) and loaded onto a Superdex 200 column equilibrated with $300 \mathrm{mM}$ Tris- $\mathrm{HCl}$. All the purification steps were performed at $4{ }^{\circ} \mathrm{C}$ and $\mathrm{pH} 7.60$.

\section{Enzyme kinetic assays}

Steady-state kinetic studies of all Fdhs were performed under an argon atmosphere at $37{ }^{\circ} \mathrm{C}$ by monitoring the benzyl viologen reduction at $555 \mathrm{~nm}\left(\varepsilon=12 \mathrm{mM}^{-1} \mathrm{~cm}^{-1}\right)$ as described by Rivas et al. [15]. For the study of the isotopic effect, sodium formate was replaced by sodium formate labeled with deuterium at the $\alpha$-position $\left(99 \%{ }^{2} \mathrm{H}\right.$, Cambridge Isotopes).

\section{Theoretical calculations}

The model chosen to study the catalytic mechanism of Fdh includes a molybdenum (or tungsten) bis(dimethyl dithiolene) complex, in which the ligands represent a portion of the pyranopterinic cofactor. The coordination sphere of the molybdenum (or tungsten) center was completed with a sulfur atom, and a $\mathrm{SeCH}_{3}$ group that was chosen to mimic the $\mathrm{SeCys}^{140}$ residue. The model was subsequently completed with the conserved His ${ }^{141}, \mathrm{Arg}^{333}$, and $\mathrm{Val}^{139}$ residues (amino acid numbering corresponding to E. coli Fdh-H) $[4,6,7,12,13]$. Conventional protonation states for all amino acids at $\mathrm{pH} 7$ were adopted and the carboxylate and the amino groups of the terminal amino acids of each chain of the model were protonated. This means that the C-terminus of each amino acid of the model was replaced by an aldehyde group and the $\mathrm{N}$-terminus was replaced by an amine group. For the calculations, the position of the atoms marked with the letter $F$ in Fig. 2 was frozen in order to keep the optimized structures close to the $\mathrm{X}$-ray structure.

Several methods are available to study chemical reactions, including static quantum approaches or even advanced sampling techniques, e.g., umbrella sampling [21], metadynamics [22-24], and parallel tempering [25]. In this work, the reaction mechanism was explored using the static quantum approach [26].

The starting coordinates were taken from the $2.80-\AA$ resolution X-ray structure of the oxidized form of $E$. coli Fdh-H (PDB ID 1FDO), where the oxygen ligand to the molybdenum ion was replaced by a sulfur atom. All geometry optimizations were performed with Gaussian 03 [27], applying DFT methods and the 6-31G(d) basis set. Becke's [28] three-parameter exchange functional was used together with the functional of Lee et al. [29] 


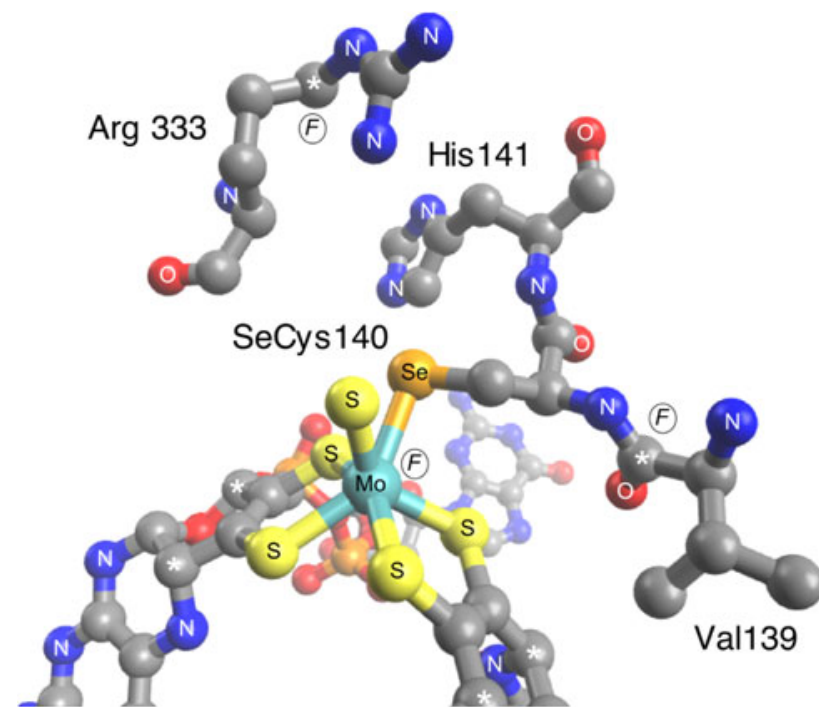

Fig. 2 Model used to study the catalytic mechanism of Fdh (frozen atoms are marked with an $F$ and the truncation of the residues is marked with a white asterisk, PDB ID 1FDO)

(B3LYP) as implemented in Gaussian [30, 31]. In all geometry optimizations, we first searched for the transition state starting from a structure similar to the reactants model. This was generally obtained with a scan where the reaction coordinate in which we were interested was shortened or stretched. Once the transition state had been located, the reactants and the products associated with it were determined through internal reaction coordinate calculations. In all cases, the geometry optimizations and the stationary points were obtained with standard Gaussian convergence criteria. The transition state structures were all verified by vibrational frequency calculations, having exactly one imaginary frequency with the correct transition vector even when frozen atoms were used, which shows that the frozen atoms are almost free from steric strain.

The final electronic energies were calculated using the all-electron $6-311++\mathrm{G}(3 \mathrm{df}, 2 \mathrm{pd})$ basis set. Zero-point corrections, thermal effects, and entropic effects $(T=$ $310.15 \mathrm{~K}, P=1$ bar) were added to all calculated energies with the $6-31 \mathrm{G}(\mathrm{d})$ basis set [26]. To estimate solvation effects of the rest of the enzyme, single-point calculations on the optimized geometries were performed with the conductor-like polarizable continuum model, as implemented in Gaussian 03 [27], with the B3LYP/6-311++G (3df,2pd) level. This feature is of particular importance in the study of enzymatic catalysis because a continuum model is normally used as an approximation of the effect of the global enzyme environment in a reaction. A dielectric constant of $\varepsilon=4$ was chosen to describe the protein environment of the active site in accordance with previous suggestions [16, 32-34]. The atomic spin-density distributions were calculated at the B3LYP level by employing a Mulliken population analysis using the $6-31 \mathrm{G}(\mathrm{d})$ basis set [35].

\section{Results}

Steady-state kinetic studies

The kinetic parameters of the three Fdhs isolated from sulfate-reducing bacteria are given in Table 1 together with those of Fdh-H isolated from E. coli K12. The $K_{\mathrm{m}}$ and $k_{\mathrm{cat}}$ values of the molybdenum- and tungsten-containing Fdh enzymes isolated from Desulfovibrio species are of the same order of magnitude, suggesting that the enzyme as a whole rather than the type of metal in the active site (molybdenum or tungsten) is responsible for the turnover rate and specificity. This is in line with DFT calculations that confirmed that changing molybdenum for tungsten in the active site model does not significantly modify the activation $\left(E_{\mathrm{a}}\right)$ and the reaction $\left(E_{\mathrm{r}}\right)$ energies of the ratelimiting step of the catalytic mechanism (not shown), and hence it will not be discussed further.

As shown in Table 1, Fdhs from sulfate-reducing bacteria catalyze deuterioformate oxidation with no significant variations of the $K_{\mathrm{m}}$ values with respect to the reactions with formate with natural isotope composition, but the $k_{\text {cat }}$ values dropped approximately $2-3$ times. This indicates that the formate $\alpha$-proton transfer to the accepting group is involved in the rate-limiting step of the catalytic mechanism, a fact which was assessed with DFT calculations as

Table 1 Kinetic parameters obtained through steady-state enzyme kinetic studies of molybdenum-containing formate dehydrogenases $(M o-F d h)$ and tungsten-containing formate dehydrogenases $(W-F d h)$

\begin{tabular}{|c|c|c|c|c|c|c|c|}
\hline & \multicolumn{2}{|l|}{$k_{\text {cat }}\left(\mathrm{s}^{-1}\right)$} & \multicolumn{2}{|l|}{$K_{\mathrm{m}}(\mu \mathrm{M})$} & \multicolumn{2}{|l|}{$k_{\mathrm{cat}} / K_{\mathrm{m}}$} & \multirow[t]{2}{*}{ Reference } \\
\hline & $\mathrm{HCOO}^{-}$ & $\mathrm{DCOO}^{-}$ & $\mathrm{HCOO}^{-}$ & $\mathrm{DCOO}^{-}$ & $\mathrm{HCOO}^{-}$ & $\mathrm{DCOO}^{-}$ & \\
\hline Desulfovibrio desulfuricans Mo-Fdh & 347 & 104 & 64 & 81 & 5.4 & 1.3 & This work \\
\hline Desulfovibrio gigas W-Fdh & 174 & 96 & 51 & 66 & 3.4 & 1.5 & This work \\
\hline Desulfovibrio alaskensis W-Fdh & 241 & 191 & 10 & 9 & 24.1 & 21.2 & This work \\
\hline Escherichia coli $\mathrm{K} 12 \mathrm{Mo}-\mathrm{Fdh}-\mathrm{H}$ & 1,367 & 1,133 & 13,300 & 49,500 & 0.1 & 0.02 & {$[36]$} \\
\hline
\end{tabular}

Enzyme kinetic plots are reported in Fig. S2. 
described later. A different behavior was observed for E. coli $\mathrm{Fdh}-\mathrm{H}$ (see kinetic parameters in Table 1), which is attributed to the presence of azide (which is necessary to preserve enzyme activity during purification) in the enzyme preparations [36]. This was corroborated by adding sodium azide to $D$. desulfuricans $F d h$ preparations in concentrations similar to that used in the E. coli Fdh-H purification, which resulted in lower $k_{\mathrm{cat}}$ values that are not affected by the substrate employed $\left({ }^{1} \mathrm{HCOO}^{-}\right.$or ${ }^{2} \mathrm{HCOO}^{-}$, not shown). This is in line with EPR results of azideincubated $D$. desulfuricans $\mathrm{Fdh}$ that showed molybdenum signals identical to those of E. coli Fdh-H [15].

\section{DFT calculations}

\section{Structure and oxidation state of the active site}

In metal coordination environments rich in sulfur ligands such as the one studied here for the molybdenum ion, it is difficult to determine the formal oxidation state of the atoms composing the model. For this reason it was assumed that each dimethyl dithiolate ligand $\left(\left[\mathrm{H}_{6} \mathrm{C}_{4} \mathrm{~S}_{2}\right]^{2-}\right)$ has a net charge of $-2, \mathrm{SeCH}_{3}$ has a charge of -1 , and the $S_{i}$ has a charge of -2 .

Since molybdenum (or tungsten) can be found in these enzymes in three different oxidation states $\left(\mathrm{Mo}^{4+}, \mathrm{Mo}^{5+}\right.$, or $\mathrm{Mo}^{6+}$ ), our first task was to determine the formal oxidation state of the metal ion in the X-ray structures of the oxidized and reduced forms of Fdh. For this purpose, the hexacoordinated model of the active site described in "Theoretical calculations" was optimized assuming formal oxidation states of $\mathrm{Mo}^{4+}$ (charge -3 , multiplicity 1 ), $\mathrm{Mo}^{5+}$ (charge -2 , multiplicity 2 ), and $\mathrm{Mo}^{6+}$ (charge -1 , multiplicity 1). The resulting structures obtained after optimization are depicted in Fig. 3.
The calculations revealed that the structures optimized assuming $\mathrm{Mo}^{4+}$ and $\mathrm{Mo}^{5+}$ ions are very similar (Fig. 3). In both cases, the selenium atom is found approximately $6.1 \AA$ from the $\mathrm{S}_{\mathrm{i}}$, which remains bound to the molybdenum ion with a bond length of approximately $2.2 \AA$. These observations are in line with the X-ray structure of the formate-reduced E. coli $\mathrm{Fdh}-\mathrm{H}$ (PDB ID 2IV2), in which the $\mathrm{SeCys}$ residue is stabilized with a neighboring arginine by hydrogen bonds $11 \AA$ from the molybdenum ion [13]. Only when the formal oxidation state $\mathrm{Mo}^{6+}$ is assumed the selenium atom remains bound to the molybdenum ion and to the $\mathrm{S}_{\mathrm{i}}$ ligand at approximately 2.9 and $2.3 \AA$, respectively (Fig. 3).

The different conformational arrangements of the SeCys residue observed in the three models can be explained taking into account the charge distribution. The total negative charge of the system is higher in the $\mathrm{Mo}^{4+}$ and $\mathrm{Mo}^{5+}$ models. A Mulliken population analysis reveals that the charge is mainly located at the selenium atom $(-0.71$ a.u. for $\mathrm{Mo}^{4+}$ and -0.67 a.u for $\left.\mathrm{Mo}^{5+}\right)$, the $\mathrm{S}_{\mathrm{i}}(-0.68$ a.u. for $\mathrm{Mo}^{4+}$ and -0.47 a.u. for $\left.\mathrm{Mo}^{5+}\right)$, and at the dimethyl dithiolenes ( -0.9 a.u. for $\mathrm{Mo}^{4+}$ and -0.4 a.u. for $\mathrm{Mo}^{5+}$ ). This charge distribution creates a repulsive environment between selenium and $\mathrm{S}_{\mathrm{i}}$ ligands that produces the dissociation of the SeCys residue. In fact, DFT calculations performed to evaluate the formation of the ES complex with the $\mathrm{Mo}^{4+}$ and $\mathrm{Mo}^{5+}$ models yielded activation energies of approximately $50 \mathrm{kcal} / \mathrm{mol}$, which rules out the catalysis starting with the enzyme in the $\mathrm{Mo}^{4+}$ and $\mathrm{Mo}^{5+}$ forms.

The Mulliken population analysis in the $\mathrm{Mo}^{6+}$ model reveals that the negative charge is distributed on the dimethyl dithiolene ligands ( -0.4 a.u.) and the molybdenum ion ( -0.4 a.u.). In contrast to the other two models, the $S_{\mathrm{i}}$ ligand is neutral $(0.05 \mathrm{a} . \mathrm{u})$, which, as will be seen
Fig. 3 Optimized structure of the active site models of Fdh assuming $\mathrm{Mo}^{4+}, \mathrm{Mo}^{5+}$, and $\mathrm{Mo}^{6+}$ formal oxidation states

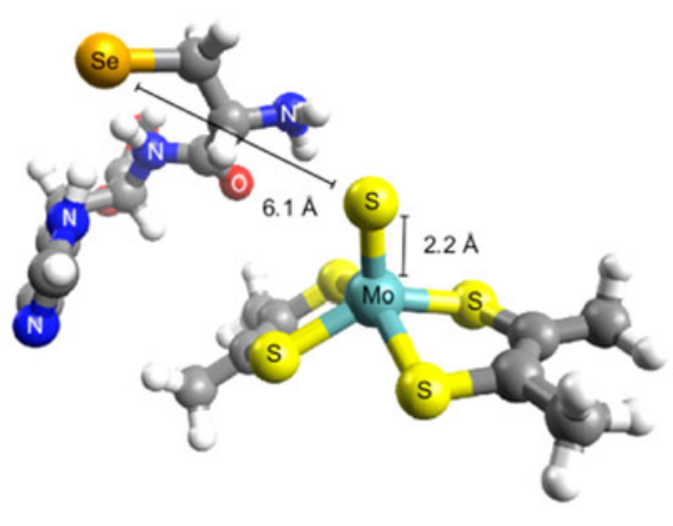

$\mathrm{Mo}(\mathrm{IV}), \mathrm{Mo}(\mathrm{V})$

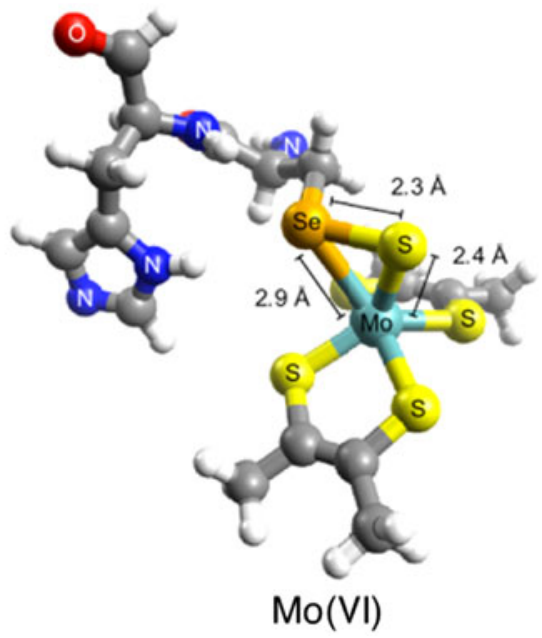


later, favors the interaction of the substrate with the active site.

The charge distribution in the three models determines the type of interaction that the $S_{i}$ ligand has with the molybdenum atom. The shorter bond length (2.2 ̊) observed in the $\mathrm{Mo}^{4+}$ and $\mathrm{Mo}^{5+}$ models suggests that the $\mathrm{S}_{\mathrm{i}}$ ligand might be double-bonded to the molybdenum, whereas in the $\mathrm{Mo}^{6+}$ model the distance $(2.4 \AA)$ suggests that the $S_{\mathrm{i}}$ ligand is single-bonded to the molybdenum ion and to the SeCys residue (Fig. 3).

\section{Formation of the ES complex: the selenium-sulfur shift}

Taking into account the results described already, one can assumed that the formal oxidation state $\mathrm{Mo}^{6+}$ represents the oxidized X-ray structure (Figs. 1a, 3). However, in the course of this study it was found that formate binds the active site only if the $\mathrm{Se}-\mathrm{S}_{\mathrm{i}}$ bond is previously broken. This is achieved by increasing the $\mathrm{Se}-\mathrm{S}_{\mathrm{i}}$ distance from 2.25 to $3.56 \AA$, which requires $15.5 \mathrm{kcal} / \mathrm{mol}$ and is endothermic by $7.5 \mathrm{kcal} / \mathrm{mol}$ (Scheme 1 ). The transition state for this reaction is characterized by one imaginary frequency at $75.72 \mathrm{i} \mathrm{cm}^{-1}$. After this rearrangement, both atoms remain bound to the molybdenum ion but with different bond lengths ( $\mathrm{Se}-\mathrm{Mo}$ bond length from 2.39 to $2.59 \AA$ 践-Mo bond length from 2.91 to $2.16 \AA$ ). At this stage, the partial interaction of the formate anion produces a redistribution of the charge in the metal complex. This is observed because the charges of the $S_{i}$ and molybdenum- bis(dimethyl dithiolene) change from -0.26 to -0.60 a.u. and from -0.62 to -0.08 a.u., respectively.

Experimental evidence from EPR spectroscopy and X-ray crystallography suggests that the formate molecule reacts with the molybdenum ion $[4,5,13,15]$. The $\mathrm{Mo}^{6+}$ model obtained in this study has, however, revealed that the Se- $\mathrm{S}_{\mathrm{i}}-$ Mo complex does not have an available binding site for the formate to bind the molybdenum, indicating that the formation of the ES complex is not a straightforward process. Our studies showed that when the oxygen of the formate molecule moves toward the $S_{i}$ ligand ( -0.53 a.u.), it pushes the $S_{i}$ ligand to the position originally occupied by the selenium atom, forcing the dissociation of the SeCys residue from the molybdenum ion (Mo-Se distance from 2.72 to $4.11 \AA$ ). In this process, $\mathrm{Arg}^{333}$ has a key role driving the formate molecule toward the $S_{i}$ ligand through two strong hydrogen bonds (1.74 $\AA$ ). Because of the repulsive environment generated by the approximation of the formate anion toward the negatively charged metallic complex, this reaction requires $20.0 \mathrm{kcal} / \mathrm{mol}$ (Scheme 2). However, this high energetic barrier is compensated as the reaction is exothermic $(-5.7 \mathrm{kcal} / \mathrm{mol})$ and ends with the formate molecule bound to the molybdenum ion by a single bond $(2.14 \AA)$. The $S_{i}$ also remains single-bonded to the molybdenum at $2.50 \AA$ and loses its negative charge ( -0.06 a.u.). At this stage, the SeCys residue dissociates from the molybdenum, but remains covalently bound to the $\mathrm{S}_{\mathrm{i}}(2.26 \AA)$.

The transition state of this reaction (characterized by one imaginary frequency of $119.62 \mathrm{i} \mathrm{cm}^{-1}$ ) clearly
Scheme 1 Enzyme-substrate (ES) interaction (the total charge and multiplicity of the system are in brackets)

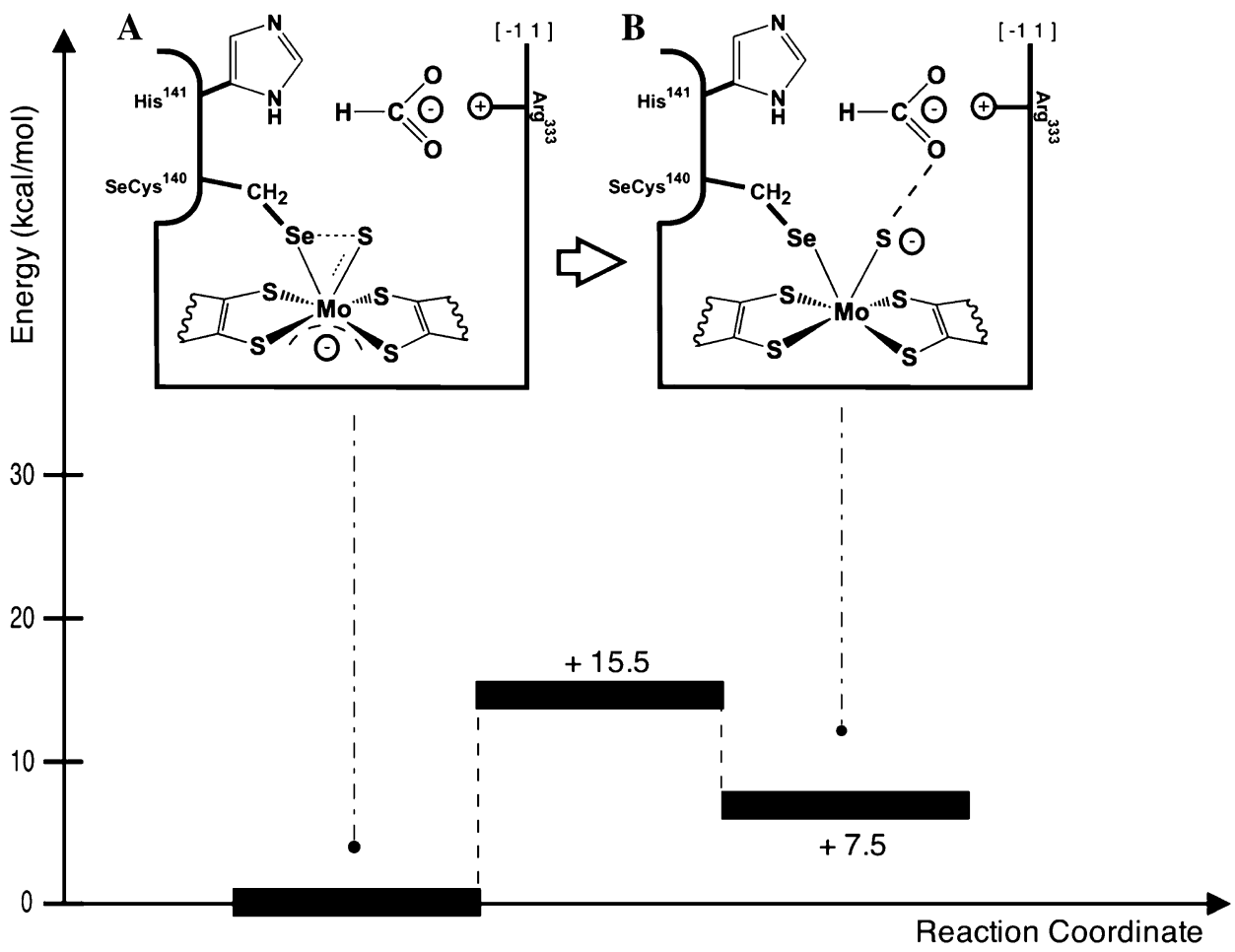


Scheme 2 Proposed seleniumsulfur shift during the formation of the ES complex (the total charge and multiplicity of the system are in brackets)

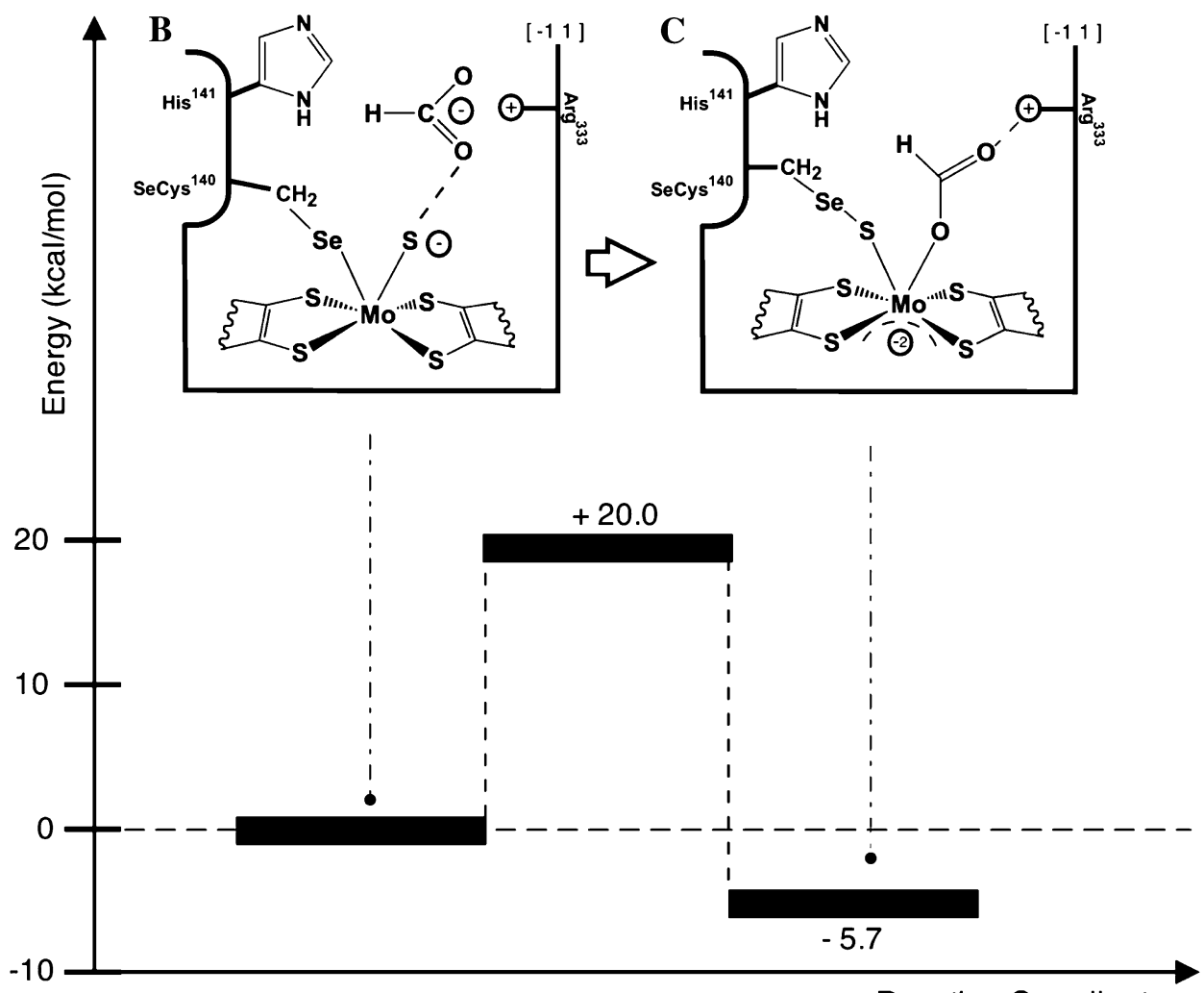

Reaction Coordinate demonstrates the feasibility of three events in one step: (1) dissociation of the SeCys residue from the molybdenum ion (selenium shift), (2) conformational rearrangement of the $S_{i}$ (sulfur shift) and, (3) coordination of the formate molecule to the molybdenum ion.

This reaction is only possible owing to the presence of the molybdenum-dithiolene complex and the conserved $\mathrm{Arg}^{333}$ that act to buffer the negative charge of the formate anion (the charge of the molybdenum-dithiolene complex changes from -0.13 to -0.72 a.u.). This type of behavior was also proposed for the catalytic mechanism of the periplasmic nitrate reductase, where the nitrate anion binds a similar negatively charged active site [37]. These results suggest that one of the roles of the pyranopterinic cofactors in these enzymes might be, in addition to providing an electron transfer pathway $[9,10]$, to cushion the electrostatic repulsion produced by the negative charge of formate, lowering the activation energy barrier for the formation of the ES complex.

\section{Selenide anion formation and formate oxidation}

The oxidation of the formate molecule to carbon dioxide involves the transfer of either a proton or a hydride from formate to a neighboring atom. Two atoms could accomplish this function: the selenium atom from the SeCys residue or the $\mathrm{S}_{\mathrm{i}}$. Our results revealed that this step is only possible with the participation of the SeCys residue. Moreover, it was observed that $\mathrm{His}^{141}$ is also very important for this reaction, since the exclusion of His ${ }^{141}$ from the model yielded an activation energy of approximately $45 \mathrm{kcal} / \mathrm{mol}$.

Computational results including $\mathrm{His}^{141}$ indicated that formate oxidation occurs via the formation of a selenide anion, which implies that the $\mathrm{Se}-\mathrm{S}_{\mathrm{i}}$ bond must be broken in a reaction that is not energetically favorable owing to the instability of the product. In this sense, the SeCys residue dissociation reaction requires $E_{\mathrm{a}}=18.4 \mathrm{kcal} / \mathrm{mol}$ and is endothermic by $16.7 \mathrm{kcal} / \mathrm{mol}$ (Scheme 3 ). In the reactants, the selenium atom is covalently bound to the $S_{i}(2.65 \AA)$, which is also bound to the molybdenum ion $(2.24 \AA)$. As the $\mathrm{Se}-\mathrm{S}_{\mathrm{i}}$ bond length is stretched, the selenium atom forms a hydrogen bond with one $\mathrm{NH}$ group of the imidazole ring of the conserved His ${ }^{141}$, which lowers $\mathrm{E}_{\mathrm{a}}$. In the optimized structure of the transition state $\left(40 \mathrm{icm}^{-1}\right)$, the selenium atom is almost dissociated from the $S_{i}(3.20 \AA)$, whereas His ${ }^{141}$ undergoes a conformational rearrangement in which the $\mathrm{NH}$ group is aligned with the selenium atom to form a hydrogen bond. In the product of the reaction, the $\mathrm{Se}-\mathrm{S}_{\mathrm{i}}$ bond is broken $(4.44 \AA$ ), and the selenide anion (with a charge of -0.83 a.u.) is formed. In this stage of the reaction the $\mathrm{NH}$ group from the histidine imidazole and the selenium atom are $2.56 \AA$ apart, indicating the important role of the conserved His ${ }^{141}$ in stabilizing the selenide anion 
Scheme 3 Selenide anion formation (the total charge and multiplicity of the system are in brackets)

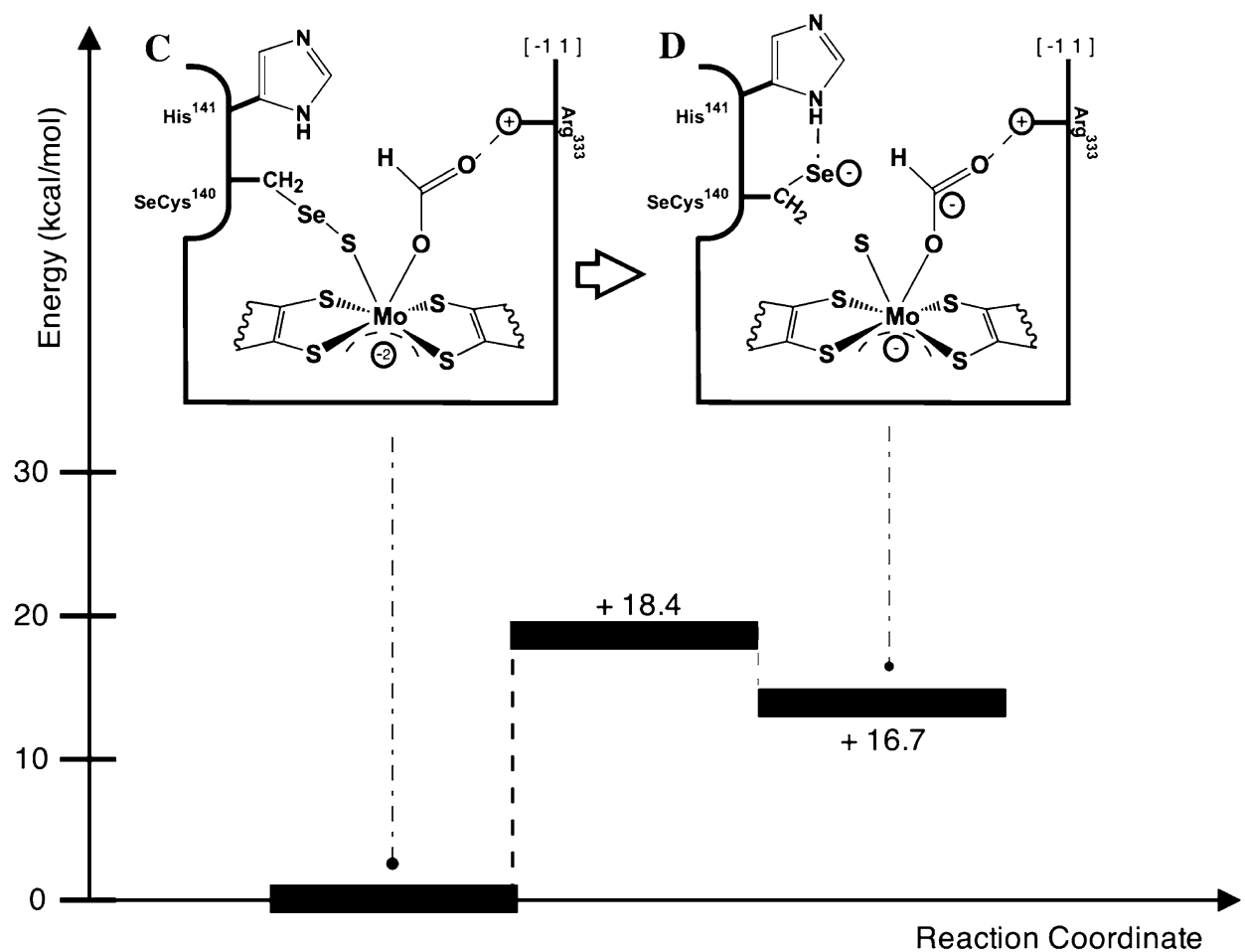

that will perform the proton abstraction. We also tested the hypothesis of the proton transfer between the formate molecule and the selenium atom in one concerted step, i.e., with the selenium atom binding the $\mathrm{S}_{\mathrm{i}}$ ligand. However, $E_{\mathrm{a}}$ values of approximately $45 \mathrm{kcal} / \mathrm{mol}$ were obtained.

The next step, which comprises the formate oxidation per se, starts with the selenide anion positioned $3.84 \AA$ from the formate hydrogen (Scheme 4). During the search for the transition state for this reaction, it became clear that when the $\mathrm{Se}-\mathrm{H}_{\text {formate }}$ distance is shortened, the $\mathrm{S}_{\mathrm{i}}-\mathrm{C}_{\text {formate }}$ distance also decreases. This is evident in the transition state of this reaction $\left(589.48 \mathrm{i} \mathrm{cm}^{-1}\right)$, in which the selenium atom is found $1.89 \AA$ from the formate hydrogen and the $S_{i}$ is found $2.15 \AA$ from the formate carbon (3.30 $\AA$ in the reactants).

The optimized structure of the transition state also revealed that a proton with charge of +0.81 a.u. is transferred to the SeCys residue, which is in agreement with the results reported by Leopoldini et al. [16]. It is possible that the $\mathrm{S}_{\mathrm{i}}$ has some role in the proton transfer between the formate and $\mathrm{SeCys}$ residue since the $\mathrm{Si}-\mathrm{H}_{\text {formate }}$ distance found in the transition state of the reaction in Scheme 4 is as low as $2.35 \AA$.

In the product of the reaction depicted in Scheme 4, the SeCys residue is protonated and moves away from the active site (Mo-Se distance $7.66 \AA$ ). Consequently, the hydrogen bond between the selenium atom and the His ${ }^{141}$ residue is broken and a new hydrogen bond between the $\mathrm{S}_{\mathrm{i}}$ and His ${ }^{141}$ is formed $(2.53 \AA)$. The carbon from the $-\mathrm{CO}_{2}$ group is now bound to the $\mathrm{S}_{\mathrm{i}}$ at a distance of $1.78 \AA$, and one of the oxygens from the $-\mathrm{CO}_{2}$ remains bound to the molybdenum ion (Fig. 4).

The proton transfer reaction requires $21.2 \mathrm{kcal} / \mathrm{mol}$ and is exothermic by $-27.7 \mathrm{kcal} / \mathrm{mol}$ (Scheme 4), indicating that despite the relatively high activation energy barrier of the two steps, this reaction is feasible. It is important to mention that a high $E_{\mathrm{a}}$ value was expected for this step owing to the primary isotopic effect observed with deuterium-labeled sodium formate in the enzyme kinetic studies mentioned before (Table 1).

\section{Carbon dioxide release}

The next step of the catalytic cycle involves the release of carbon dioxide and the two-electron oxidation of the active site. This can be accomplished in two different ways: (1) the carbon dioxide is released before the oxidation of the active site or (2) the active site is oxidized to promote the product release.

In the first case, the dissociation of the $\mathrm{CO}_{2}$ requires the two steps depicted in Scheme 5. Firstly, the Mo-O bond is broken (Mo-O distance changes from 2.21 to $3.81 \AA$ ), producing an intermediate stage where the $\mathrm{CO}_{2}$ still remains attached to the active site through the $\mathrm{S}_{\mathrm{i}}-\mathrm{C}$ bond. After the break of the Mo-O bond, the Mo- $\mathrm{S}_{\mathrm{i}}$ bond length decreases from 2.54 to $2.34 \AA$. The charge distribution is conserved during the reaction, being mainly localized on the dimethyl dithiolene ligands ( -1.33 a.u.) and around the $-\mathrm{CO}_{2}$ group $\left(-0.75\right.$ a.u.). This reaction needs $E_{\mathrm{a}}=13.3 \mathrm{kcal} / \mathrm{mol}$, is 
Scheme 4 Formate oxidation and $\mathrm{C}-\mathrm{H}$ bond cleavage (the total charge and multiplicity of the system are in brackets)

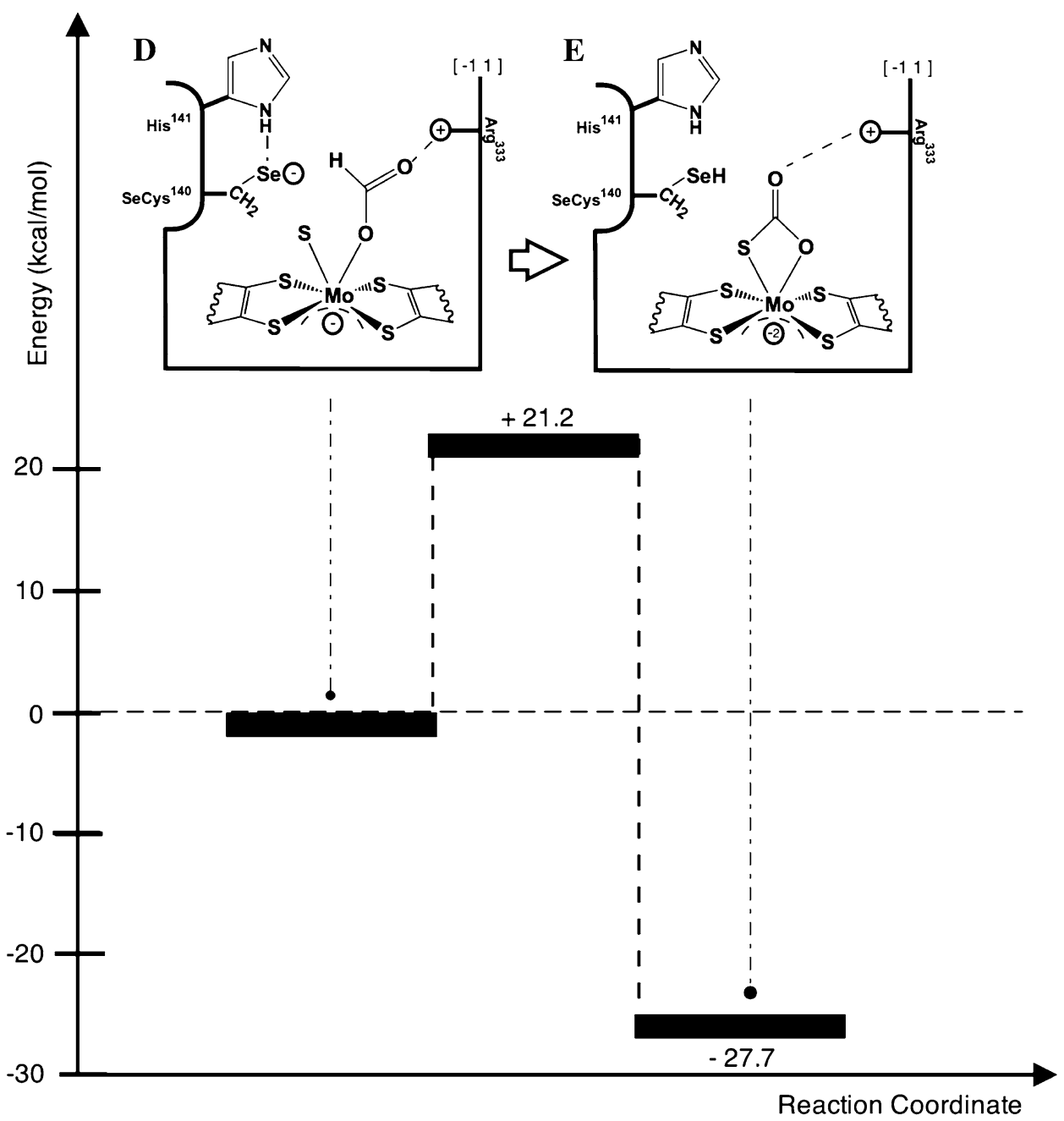

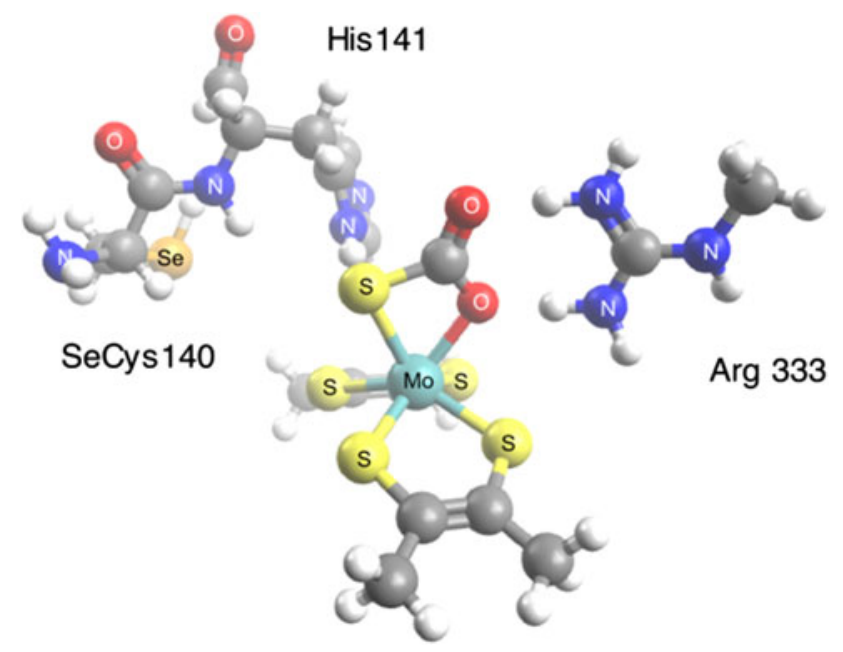

Fig. 4 Product obtained after the proton transfer reaction from formate to $\mathrm{SeCys}{ }^{140}$

endothermic $\left(E_{\mathrm{r}}=7.2 \mathrm{kcal} / \mathrm{mol}\right)$, and yielded a transition state characterized by one imaginary frequency of $67 \mathrm{i} \mathrm{cm}^{-1}$.
To complete the product release, the $\mathrm{S}_{\mathrm{i}}-\mathrm{C}$ bond must be broken. This happens when the $\mathrm{S}_{\mathrm{i}}-\mathrm{C}_{\text {formate }}$ distance increases from $1.89 \AA$ in the reactants to $2.20 \AA$ in the transition state $\left(123 \mathrm{i} \mathrm{cm}^{-1}\right)$. In the product, the $\mathrm{CO}_{2}$ molecule is found at $3.81 \AA\left(\mathrm{S}_{\mathrm{i}}-\mathrm{C}\right.$ distance $)$ as a neutral molecule (charge -0.05 a.u.). This reaction yielded $E_{\mathrm{a}}=4.5 \mathrm{kcal} / \mathrm{mol}$ and $E_{\mathrm{r}}=-7.8 \mathrm{kcal} / \mathrm{mol}$, indicating the two-step process of $\mathrm{CO}_{2}$ release is almost thermoneutral $(-0.6 \mathrm{kcal} / \mathrm{mol})$.

In the second case (not shown), it was assumed that after the transfer of the proton of the formate to the SeCys residue, two electrons would be immediately transferred to the redox centers included in the electron transfer pathway of the protein [9, 10]. This implies the two-electron oxidation of the active site, for which it was necessary to manually adjust the charge of the model to +1 . Compared with the model previous to these modifications, the only changes are the $\mathrm{Mo}-\mathrm{S}_{\mathrm{i}}$ and $\mathrm{Mo}-\mathrm{O}_{\text {formate }}$ bond lengths, decreasing from 2.54 to $2.39 \AA$ and from 2.21 to $2.15 \AA$, respectively. The negative charge remains located in the $-\mathrm{CO}_{2}$ group, whereas the dithiolene ligands become 


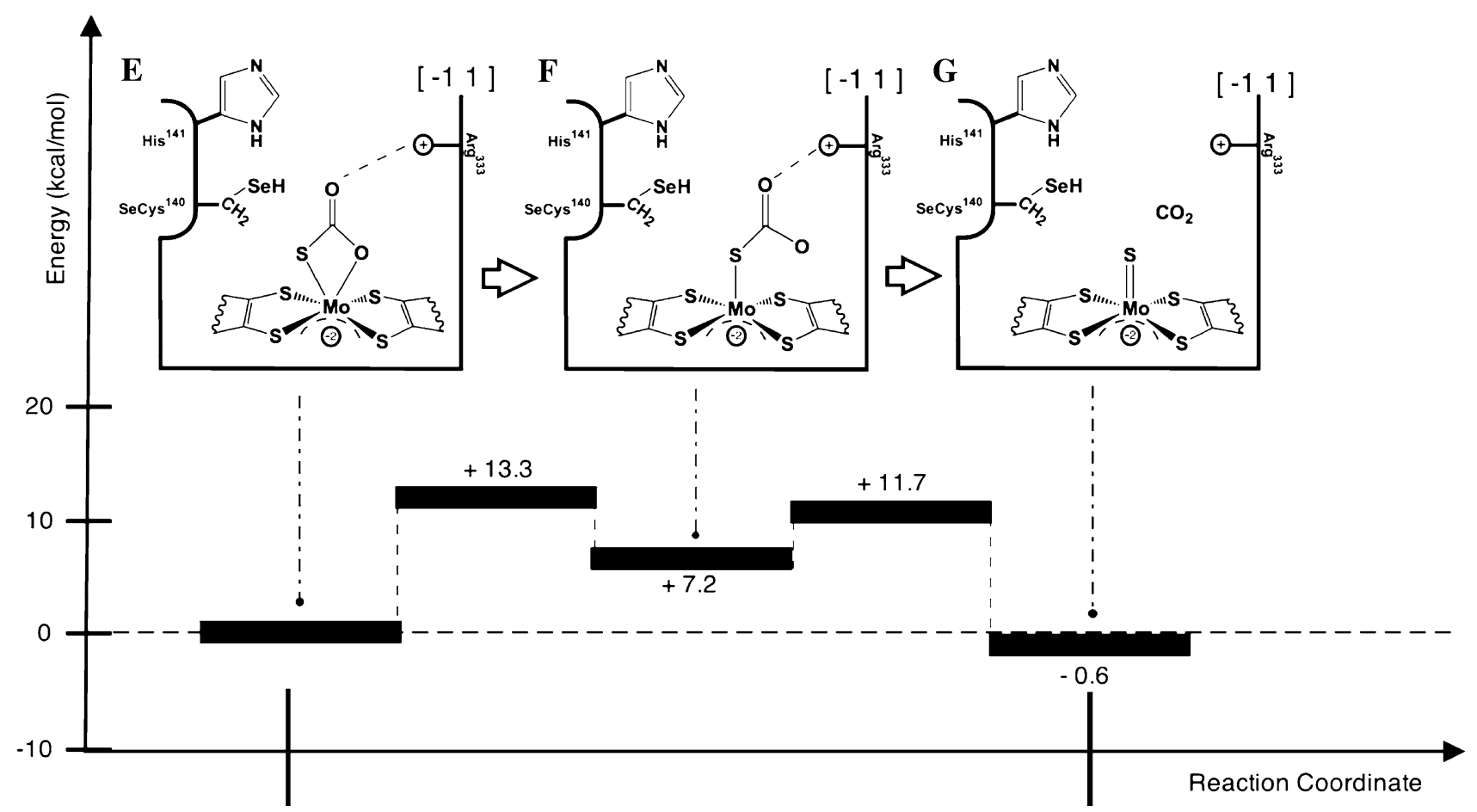

Scheme 5 Carbon dioxide release mechanism preceding the oxidation of the active site (the total charge and multiplicity of the system are in brackets)

positively charged. In the transition state (imaginary frequency of $127.91 \mathrm{i} \mathrm{cm}^{-1}$ ) it can be noted that both the Mo-O and the $\mathrm{S}_{\mathrm{i}}-\mathrm{C}$ bonds are simultaneously broken at the same time as the Mo- $\mathrm{S}_{\mathrm{i}}$ bond length is stretched to $2.12 \AA$. In the products of this reaction, the dissociation of the $\mathrm{CO}_{2}$ molecule is accomplished and similarly to what happens in the first hypothesis, the $S_{i}$ becomes double-bonded to the molybdenum. This reaction requires an activation energy of $25.6 \mathrm{kcal} / \mathrm{mol}$ and is exothermic by $-16.6 \mathrm{kcal} / \mathrm{mol}$.

Analyzing the activation energy profiles for the two hypotheses tested for $\mathrm{CO}_{2}$ release, we found that the first hypothesis emerges as more probable, even though it needs two steps to release the product.

\section{Regeneration of the active site to the initial state}

On the basis of the complex obtained after $\mathrm{CO}_{2}$ release, the last steps of the proposed catalytic mechanism will involve the transfer of the proton attached to the SeCys residue, oxidation of the active site from Mo(IV) to Mo(VI), and binding of the SeCys residue to the molybdenum ion. The proton transfer process could be assisted by solvent molecules or by some neighboring amino acid residues. However, because there are no experimental data about how this is accomplished, the only thing that we were able to model was the binding of the resulting anionic SeCys residue to the $\mathrm{Mo}(\mathrm{VI})$ ion in the oxidized active site. In this sense, it is expected that the loop in which the
SeCys residue is located undergoes a conformational rearrangement.

To model this reaction, the proton of the SeCys residue was manually deleted and the charge of the system was adjusted to obtain a molybdenum ion in the +6 formal oxidation state to emulate the reoxidation of the active site. As expected, the overall charge of the active site became more positive. The charge of the $S_{i}$ ligand changed from -0.63 to 0.19 a.u. without $\mathrm{Mo}-\mathrm{S}_{\mathrm{i}}$ bond length modification (from 2.15 to $2.14 \AA$ ). The anionic selenium, with a charge of -0.71 a.u., is $3.52 \AA$ from the $S_{i}$ ligand and interacts very closely with $\mathrm{His}^{141}$ (2.64 $\AA$ ) through a hydrogen bond with the imidazole NH group. From this point, two pathways that will depend on the presence or absence of a substrate molecule near the active site can be drawn. For the first pathway, the active site structure in the absence of formate returns to the initial state, in which both the selenium atom and the $S_{i}$ ligand are bound to the Mo(VI) ion. The computational results indicate that this process requires two steps and is energetically feasible (Scheme 6). The first step implies the binding of the selenium atom to the $S_{i}$ ligand. This process yielded $E_{\mathrm{a}}=2 \mathrm{kcal} / \mathrm{mol}$ and is exothermic by $-18 \mathrm{kcal} / \mathrm{mol}$. The optimized geometry of the transition state (imaginary frequency of $33 \mathrm{i} \mathrm{cm}^{-1}$ ) reveals that the selenium atom is $2.25 \AA$ from the $\mathrm{S}_{\mathrm{i}}$ ligand and $3.35 \AA$ from the molybdenum ion. In the product of this reaction, the Mo- $\mathrm{S}_{\mathrm{i}}$ bond length increases from 2.14 to $2.35 \AA$, indicating that the Mo- $\mathrm{S}_{\mathrm{i}}$ double bond become a single bond upon 


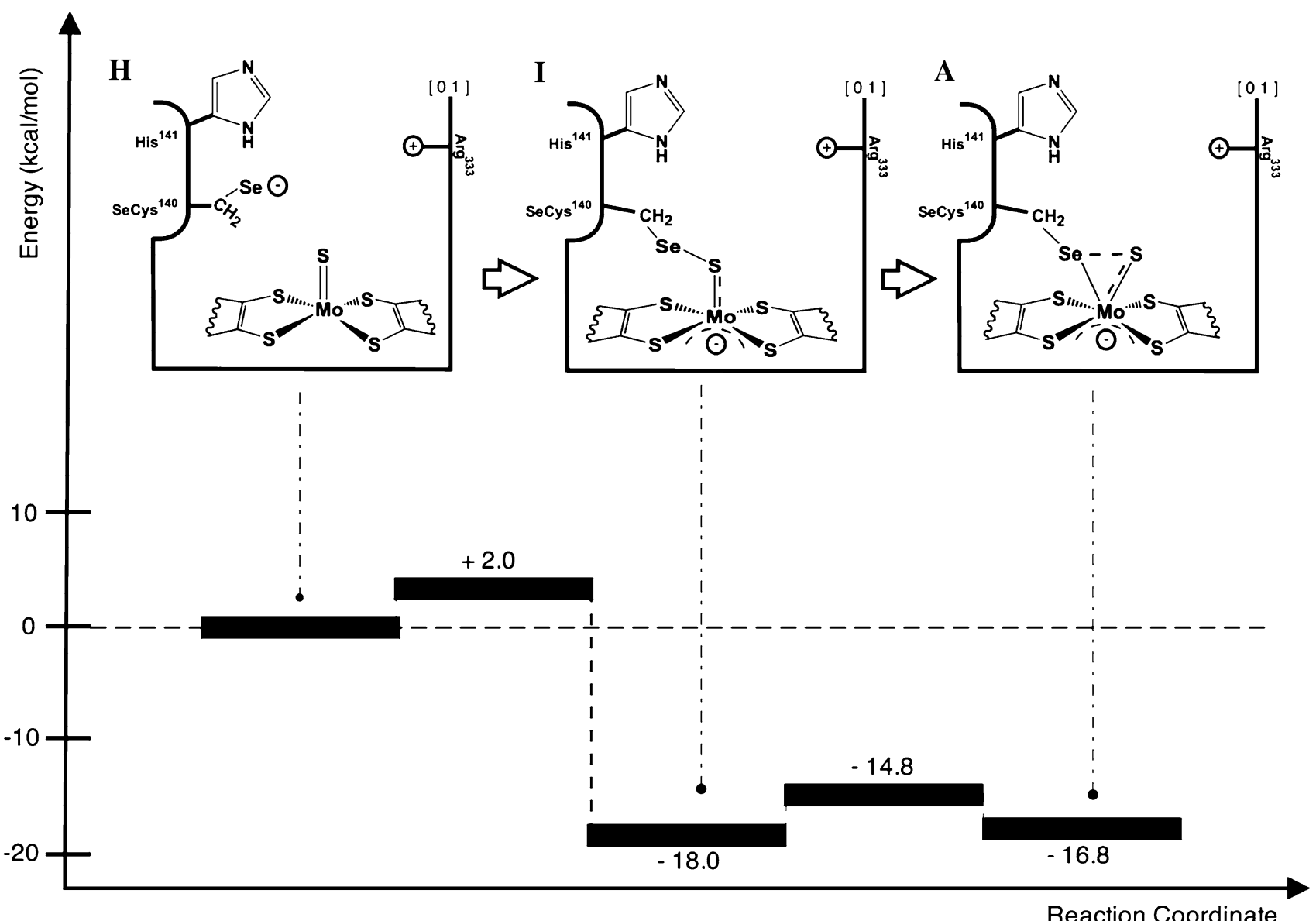

Scheme 6 Regeneration of the active site to the initial oxidized state (the total charge and multiplicity of the system are in brackets)

selenium atom binding. In spite of this rearrangement, the selenium atom does not bind the molybdenum ion, remaining $3.94 \AA$ away. The charge distribution remains almost unaltered $(-0.67$ a.u. in each dithiolene ligand, -0.34 a.u. in the $S_{i}$, and -0.24 a.u. in the selenium). After $\mathrm{Se}-\mathrm{S}_{\mathrm{i}}$ bond formation, the SeCys residue binds the Mo(VI) ion and the $\mathrm{S}_{\mathrm{i}}$ as in the initial state. The calculations yielded $E_{\mathrm{a}}=3.2 \mathrm{kcal} / \mathrm{mol}$ and $E_{\mathrm{r}}=1.2 \mathrm{kcal} / \mathrm{mol}$. The optimized geometry of the transition state (imaginary frequency of $37.68 \mathrm{i} \mathrm{cm}^{-1}$ ) shows that the selenium atom approaches the molybdenum ion (from 3.94 to $3.35 \AA$ ) and that the charge distribution and the Mo- $\mathrm{S}_{\mathrm{i}}$ and $\mathrm{Se}-\mathrm{S}_{\mathrm{i}}$ bond lengths remain unaltered. In the product, the SeCys residue binds the molybdenum ion and the $\mathrm{S}_{\mathrm{i}}$ ligand through single bonds (2.96 and $2.26 \AA$, respectively).

The second pathway is summarized in Scheme 7. The computational results showed that binding of a new formate molecule to the oxidized active site (as found in the starting species in Scheme 6) is a one-step process requiring only $E_{\mathrm{a}}=6.1 \mathrm{kcal} / \mathrm{mol}$ and $E_{\mathrm{r}}=2.7 \mathrm{kcal} / \mathrm{mol}$ (Scheme 7). In the transition state (imaginary frequency of $45 \mathrm{i} \mathrm{cm}^{-1}$ ) the Mo- $S_{\mathrm{i}}$ bond length is unaltered but the $\mathrm{S}_{\mathrm{i}}$ ligand is pushed aside, creating space for the incoming formate molecule
(Mo- $\mathrm{O}_{\text {formate }}$ bond length $3.03 \AA$ ). In the product of the reaction the formate binds the molybdenum ion $\left(\mathrm{Mo}-\mathrm{O}_{\text {formate }}\right.$ bond length $2.21 \AA$ ) and the $\mathrm{S}_{\mathrm{i}}$ ligand gains negative charge ( -0.62 a.u.). Similar to what was observed before, the conserved $\mathrm{Arg}^{333}$ has a key role in this reaction driving and stabilizing the formate anion by hydrogen bonds. However, there is a striking difference when the formate anion binds the active site in the situation presented in Scheme 7 (SeCys residue not bound to the $S_{i}$ ligand), as the energy cost of binding a new substrate molecule is $14 \mathrm{kcal} / \mathrm{mol}$ less than that obtained with the SeCys bound to the $\mathrm{S}_{\mathrm{i}}$ ligand (Scheme 2). This difference, which is attributed to the different charge distribution and steric hindrance of the complex when the SeCys residue binds the $S_{i}$ ligand, indicates that the catalytic cycle should follow this pathway instead of regenerating the active site as in the initial state.

\section{Discussion}

Scheme 8 summarizes the main conclusions on the general catalytic mechanism of metal-dependent Fdh evaluated through DFT tools. The first part of this study demonstrated 
Scheme 7 Formate binding to an intermediate species produced after oxidation of the active site (the total charge and multiplicity of the system are in brackets)

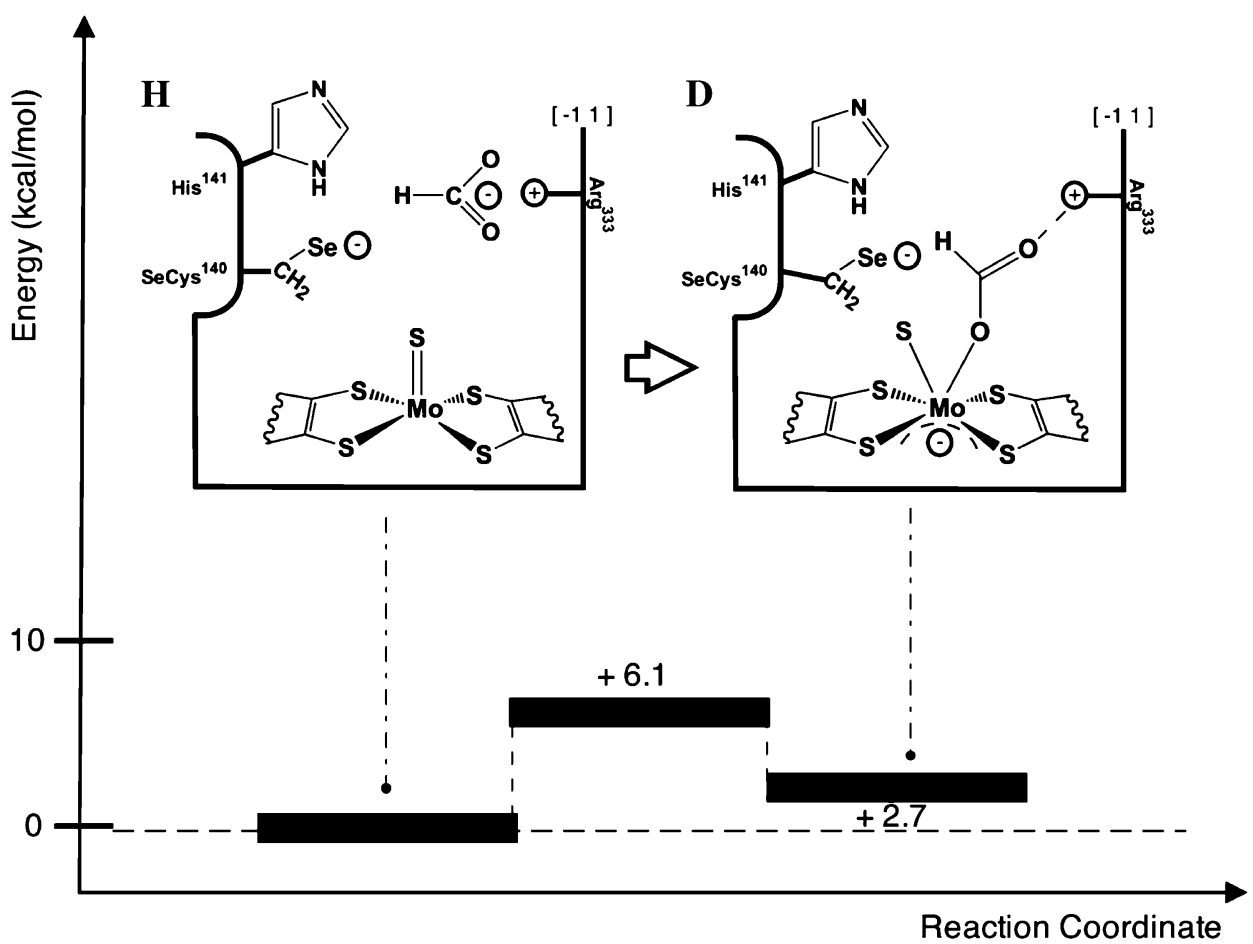

that the hexacoordinated molybdenum ion of the crystallographic structure is in the +6 oxidation state (Fig. 3a, Scheme 8, state A). Previous studies of D. desulfuricans Fdh demonstrated that this state represents an inactive form of the enzyme resulting from the aerobic purification procedure of an enzyme that works physiologically under strict anaerobic conditions [15]. This is corroborated with all the Fdh enzymes studied in this work, as they needed to be incubated with the substrate prior to electron acceptor addition to obtain their maximal specific activity (Fig. S1). This activation step implies that the enzyme performs at least one complete turnover returning to a Mo(VI) state (Scheme 8, state H) different from the initial one (Scheme 8, state A). Then, the reaction proceeds with the formate molecule binding the active site in a state where the Mo(VI) ion has an available binding position (Scheme 8, step H-D). This is indicated by the results summarized in Scheme 7, where the binding of the formate to the pentacoordinated $\mathrm{Mo}(\mathrm{VI})$ ion has $E_{\mathrm{a}}=6.1 \mathrm{kcal} / \mathrm{mol}$. In summary, the three initial steps with high activation energies (Scheme 8, steps A-B, B-C, and C-D, $E_{\mathrm{a}}=15.5$, 20.0 , and $18.4 \mathrm{kcal} / \mathrm{mol}$, respectively) represent the activation route necessary to perform the first cycle of the catalytic mechanism starting from the aerobically inactive enzyme. Step B-C in Scheme 8, which involves the process identified as a sulfur shift, is similar to that proposed for the catalytic mechanism of periplasmic nitrate reductase where the role of the SeCys residue is performed by a cysteine residue $[37,38]$. This may indicate that the sulfur shift could be a common mechanism by which these enzymes enable a free coordinating position on the metal ion for substrate coordination.

The formate oxidation process also involves the participation of amino acids that are conserved in all metaldependent Fdhs characterized so far. Our DFT calculations indicate that the side chain of the arginine residue interacts with both the substrate and the product of the reaction, guiding the substrate to the active site, stabilizing it, and driving the product release. In this sense, the positively charged side chain of arginine cancels the negative charge of the formate anion, facilitating its interaction with the active site.

One of the most intriguing roles to unveil was that of the conserved histidine residue. Khangulov et al. [5] have proposed that this residue might be involved in the deprotonation of the SeCys following the proton transfer reaction from the substrate to the selenide anion. In contrast, our calculations indicate that an $\mathrm{NH}$ group of the imidazole ring of histidine stabilizes the selenide anion through hydrogen bonds (Scheme 3). This role is also supported by the fact that, when the conserved histidine is not included in the model, the formation of the selenide anion yields an unstable transition state which results in a very high activation energy $\left(E_{\mathrm{a}} \sim 45 \mathrm{kcal} / \mathrm{mol}\right)$. These results also suggest why metal-dependent Fdhs contain a SeCys residue instead of a regular cysteine residue, as found in periplasmic nitrate reductases. The SeCys and cysteine side chains have $\mathrm{pK}_{\mathrm{a}}$ values of 5.2 and 8.2, respectively. This indicates that under physiological conditions ( $\mathrm{pH} \sim 7.00$ ) the selenium atom of the SeCys residue 

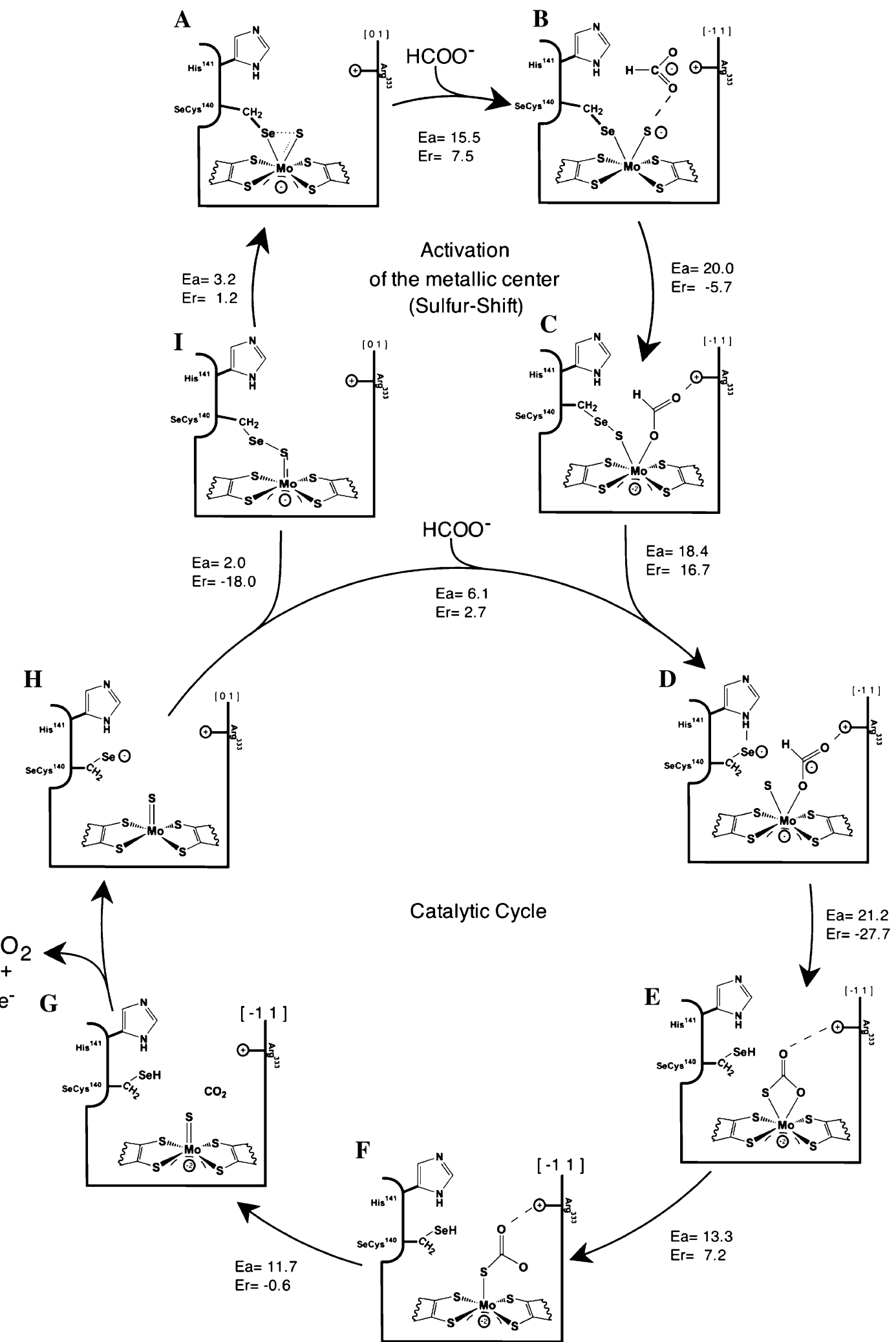

Scheme 8 Global view of the catalytic mechanism of metal-dependent formate dehydrogenase $\left(E_{\mathrm{a}}\right.$ and $E_{\mathrm{r}}$ in kilocalories per mole) 
can be found in the anionic form after breaking of the bond with the $S_{i}$ ligand bound to the molybdenum ion. Owing to the high $\mathrm{pK}_{\mathrm{a}}$ of the side chain of a cysteine residue, the presence of a stable cysteinate at physiological $\mathrm{pH}$ is unlikely. This statement has strong experimental support from early studies performed in $E$. coli $\mathrm{Fdh}-\mathrm{H}$, in which it was demonstrated that mutating the SeCys to a cysteine almost inactivates the enzyme, lowering the $k_{\text {cat }}$ by more than 2 orders of magnitude (from 2,800 to $9 s^{-1}$ ) [39].

The kinetic studies of the enzyme with the isotopically enriched ${ }^{2} \mathrm{HCOONa}$ clearly indicate that the $\mathrm{C}-\mathrm{H}$ bond break is involved in the rate-limiting step of the catalytic mechanism. Analyzing the activation energy values for every step of the simulated mechanism, and having in mind that the three initial steps of the mechanism (Scheme 8, steps A-B, $\mathrm{B}-\mathrm{C}$, and $\mathrm{C}-\mathrm{D})$ do not take part in the enzymatic catalytic cycle, we can note that the highest activation energy barrier involves the transfer of the proton from the substrate to the selenium (21.2 kcal/mol, Scheme 8, step D-E), suggesting that, in agreement with the experimental results, this is the rate-limiting event of the catalytic mechanism.

Comparing metal-dependent Fdhs and eukaryotic $\mathrm{NAD}^{+}$-dependent Fdhs, a key difference is evident in the enzymatic mechanism of formate oxidation to carbon dioxide. Metal-dependent Fdhs catalyze the transfer of a proton from the substrate to the enzyme, whereas, according to the currently accepted mechanism, $\mathrm{NAD}^{+}$-dependent Fdhs catalyze the transfer of a hydride to the $\mathrm{NAD}^{+}$ cofactor to produce an NADH [40]. Our results indicate that this fundamental difference results from the nature of the group that accepts the hydrogen ion from the substrate, i.e., a nucleophilic selenide anion versus a $\mathrm{NAD}^{+}$cation.

Acknowledgments C.S.M. thanks Fundação para a Ciência e a Tecnologia for funding (grant SFRH/BD/32478/2006). P.J.G. and N.M.F.S.A.C. thank Programa Ciência 2007 and 2008 of Fundação para a Ciência e a Tecnologia. This work was supported by projects PDCT/QUI/57701/2004 and PTDC/QUI/67052/2006 in Portugal and CAID-UNL, CONICET, and SEPCYT in Argentina. C.D.B. thanks to CONICET (Argentina).

\section{References}

1. Alberty RA (2001) Arch Biochem Biophys 389:94-109

2. Heidelberg JF, Seshadri R, Haveman SA, Hemme CL, Paulsen IT, Kolonay JF, Eisen JA, Ward N, Methe B, Brinkac LM, Daugherty SC, Deboy RT, Dodson RJ, Durkin AS, Madupu R, Nelson WC, Sullivan SA, Fouts D, Haft DH, Selengut J, Peterson JD, Davidsen TM, Zafar N, Zhou L, Radune D, Dimitrov G, Hance M, Tran K, Khouri H, Gill J, Utterback TR, Feldblyum TV, Wall JD, Voordouw G, Fraser CM (2004) Nat Biotechnol 22:554-559

3. Richardson DJ (2000) Microbiology 146(3):551-571

4. Boyington JC, Gladyshev VN, Khangulov SV, Stadtman TC, Sun PD (1997) Science 275:1305-1308
5. Khangulov SV, Gladyshev VN, Dismukes GC, Stadtman TC (1998) Biochemistry 37:3518-3528

6. Jormakka M, Tornroth S, Byrne B, Iwata S (2002) Science 295:1863-1868

7. Jormakka M, Tornroth S, Abramson J, Byrne B, Iwata S (2002) Acta Crystallogr D Biol Crystallogr 58:160-162

8. Gladyshev VN, Boyington JC, Khangulov SV, Grahame DA, Stadtman TC, Sun PD (1996) J Biol Chem 271:8095-8100

9. Moura JJ, Brondino CD, Trincao J, Romao MJ (2004) J Biol Inorg Chem 9:791-799

10. Brondino CD, Rivas MG, Romao MJ, Moura JJ, Moura I (2006) Acc Chem Res 39:788-796

11. Raaijmakers H, Teixeira S, Dias JM, Almendra MJ, Brondino CD, Moura I, Moura JJ, Romao MJ (2001) J Biol Inorg Chem 6:398-404

12. Raaijmakers H, Macieira S, Dias JM, Teixeira S, Bursakov S, Huber R, Moura JJ, Moura I, Romao MJ (2002) Structure 10:1261-1272

13. Raaijmakers HC, Romao MJ (2006) J Biol Inorg Chem 11:849-854

14. Costa C, Teixeira M, LeGall J, Moura JJG, Moura I (1997) J Biol Chem 2:198-208

15. Rivas MG, Gonzalez PJ, Brondino CD, Moura JJ, Moura I (2007) J Inorg Biochem 101:1617-1622

16. Leopoldini M, Chiodo SG, Toscano M, Russo N (2008) Chemistry $14: 8674-8681$

17. Liu MC, Peck HD Jr (1981) J Biol Chem 256:13159-13164

18. Legall J, Mazza G, Dragoni N (1965) Biochim Biophys Acta 99:385-387

19. Mota CS, Valette O, Gonzalez PJ, Brondino CD, Moura JJG, Moura I, Dolla A, Rivas MG (2010) J Bacteriol 193:2917-2923

20. Almendra MJ, Brondino CD, Gavel O, Pereira AS, Tavares $P$, Bursakov S, Duarte R, Caldeira J, Moura JJ, Moura I (1999) Biochemistry 38:16366-16372

21. Roux B (1995) Comput Phys Commun 91:275-282

22. Ensing B, De Vivo M, Liu Z, Moore P, Klein ML (2006) Acc Chem Res 39:73-81

23. Huber T, Torda AE, van Gunsteren WF (1994) J Comput Aided Mol Des 8:695-708

24. Laio A, Parrinello M (2002) Proc Natl Acad Sci USA 99:12562_ 12566

25. Berg BA, Neuhaus T (1991) Phys Lett B 267:249-253

26. Ramos MJ, Fernandes PA (2008) Acc Chem Res 41:689-698

27. Castillo R, Oliva M, Marti S, Moliner V (2008) J Phys Chem B 112:10012-10022

28. Becke AD (1993) J Chem Phys 98:5648-5652

29. Lee CT, Yang WT, Parr RG (1988) Phys Rev B 37:785-789

30. Stephens PJ, Devlin FJ, Chabalowski CF, Frisch MJ (1994) J Phys Chem 98:11623-11627

31. Vosko SH, Wilk L, Nusair M (1980) Can J Phys 58:1200-1211

32. Cerqueira NM, Fernandes PA, Eriksson LA, Ramos MJ (2006) Biophys J 90:2109-2119

33. Cerqueira NMFSA, Fernandes PA, Eriksson LA, Ramos MJ (2004) J Mol Struct Theochem 709:53-65

34. Himo F (2006) Theor Chem Acc 116:232-240

35. Cerqueira NMFSA, Fernandes PA, Ramos MJ (2011) J Chem Theory Comput 7:1356-1368

36. Axley MJ, Grahame DA (1991) J Biol Chem 266:13731-13736

37. Cerqueira NM, Gonzalez PJ, Brondino CD, Romao MJ, Romao CC, Moura I, Moura JJ (2009) J Comput Chem 30:2466-2484

38. Najmudin S, Gonzalez PJ, Trincao J, Coelho C, Mukhopadhyay A, Cerqueira NM, Romao CC, Moura I, Moura JJ, Brondino CD, Romao MJ (2008) J Biol Inorg Chem 13:737-753

39. Axley MJ, Bock A, Stadtman TC (1991) Proc Natl Acad Sci USA 88:8450-8454

40. Castillo R, Oliva M, Marti S, Moliner V (2008) J Phys Chem B 112:10012-10022 